\title{
INTRINSIC CURVATURE: A MARKER OF MILLIMETER-SCALE TANGENTIAL CORTICO-CORTICAL CONNECTIVITY?
}

\author{
LISA RONAN* \\ Brain Mapping Unit, Department of Psychiatry \\ University of Cambridge, Cambridge, UK \\ lr344@cam.ac.uk \\ RUDOLPH PIENAAR \\ Children's Hospital Boston \\ Massachusetts General Hospital, Boston, MA, USA \\ GUY WILLIAMS \\ Wolfson Brain Imaging Centre \\ University of Cambridge, UK \\ ED BULLMORE \\ Brain Mapping Unit, Department of Psychiatry \\ University of Cambridge, Cambridge, UK \\ TIM J. CROW \\ Warneford Hospital, Department of Psychiatry \\ University of Oxford, Oxford, UK \\ NEIL ROBERTS \\ Clinical Research Imaging Centre \\ Queen's Medical Research Institute \\ University of Edinburgh, Edinburgh, UK \\ PETER B. JONES \\ Behavioural and Clinical Neuroscience Institute \\ Department of Experimental Psychology \\ University of Cambridge, Cambridge, UK \\ JOHN SUCKLING \\ Department of Psychiatry \\ University of Cambridge, Cambridge, UK \\ PAUL C. FLETCHER* \\ Brain Mapping Unit, Department of Psychiatry \\ University of Cambridge, Cambridge, UK \\ pcf22@cam.ac.uk
}

\begin{abstract}
In this paper, we draw a link between cortical intrinsic curvature and the distributions of tangential connection lengths. We suggest that differential rates of surface expansion not only lead to intrinsic curvature of the cortical sheet, but also to differential inter-neuronal spacing. We propose that there follows a consequential change in the profile of neuronal connections: specifically an enhancement of the tendency towards proportionately more short connections. Thus, the degree of cortical intrinsic curvature may have implications for short-range connectivity.
\end{abstract}

Keywords: Intrinsic curvature; cortical connectivity; surface morphology.

\footnotetext{
*Corresponding authors.
} 


\section{Introduction}

In comparison to shrews, humans have a sixfold increase in cortical thickness, but a 1,700-fold increase in cortical surface area. ${ }^{1}$ This expansion is driven by an increase in the number of functional units, rather than an increase in the complexity of those units, ${ }^{2}$ and results in an increase in the degree of folding of the cortex (gyrification) to accommodate a large surface area within the volume of the cranium. At a cellular level, expansion is commensurate with a decrease in neuronal density and an increase in inter-cellular spacing. ${ }^{3,4}$ In this paper we consider the implications of this observation in terms of how differential rates of cortical expansion lead to altered neuronal spacing and connectivity. Given that such differential expansion inevitably leads to intrinsic curvature of the cortical surface, we suggest that, in view of this relationship, measurements of intrinsic curvature may offer insights to small-scale connectivity.

To start, it is worth considering certain principles of cortical connectivity. In diverse systems from C. Elegans,${ }^{5}$ to macaque visual cortex,${ }^{6}$ a distancedependent distribution of connection lengths has been demonstrated: namely a large peak of short connections and a flatter tail representing longer connections. This is essentially the characteristic of small-world architecture, ${ }^{5,7}$ which has been repeatedly shown in humans using a range of functional and morphological brain data (fMRI, EEG, cortical thickness, DTI, tract-tracing) at many levels, from brain-wide networks, ${ }^{8-10}$ through to the wiring at a neuronal level. ${ }^{11}$ Small-world networks are characterized by densely clustered local connections, in combination with relatively sparse longerrange projections. ${ }^{5,7}$ This type of arrangement is highly efficient, supporting both local specialization, as well as system-wide integration.

The distribution of neurons in the cortex is not perfectly crystalline, however if this non-uniform spatial distribution is further augmented in some way, it will lead to an additional increase in the proportion of short-term connections beyond the default preponderance described above, i.e. the distribution of connection lengths is additionally skewed due to the increase in the unevenness of the spatial distribution of neurons. A non-uniform distribution of neurons might arise either as a result of non-uniform neuronal proliferation, or differential surface expansion.

That differential expansion may result in an increase in the proportion of short connections may be explained by considering a simple case of points on a line. As the points undergo first uniform, then differential expansion, we can intuit how the histogram of distances between the points changes. In the case of the former, under uniform expansion, the distance between points remains constant. For example, if the points on the line are evenly spaced, the initial histogram of distances between points will consist of a single bin representing all points. Under uniform expansion the position of the bin will increase along the length-scale, however all points will remain in the same bin. Alternatively if we had the starting case where $50 \%$ of points were twice as close together as the rest, the initial histogram of distances would consist of two bins of equal sizes. Under uniform expansion, the position of the bins would change in a consistent way, but their proportions would not be altered.

Now consider the case of non-uniform, or differential expansion. This means that some points expand faster then others, i.e. expansion introduces a variance in to the distance between points. Importantly this variance increases as expansion increases, such that points that expand the fastest will increase the distance between them at a greater rate than points that expand the slowest. For the histogram this means that at the upper-end of the length-scale, there will be fewer and fewer points covering these longer distances. Hence even if we start with a uniform distribution of points, under differential expansion, the histogram of distances between points will become ever-more skewed to shorter distances.

We note that these are general principles of differential expansion. Although we have considered here the straightforward case of expansion of points on a line, the principle may be equally applied to points on a surface. In the next section we consider how differential expansion impacts on cortical morphology with the aim of showing how measures of morphology have implications for underlying connection length distributions.

Differential expansion of a surface may give rise to intrinsic curvature. Intrinsic curvature may be visualized thus: as the surface grows, its tangential expansion may develop in three ways: if a surface 


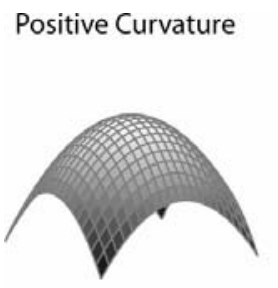

Increased Differential Growth at Centre

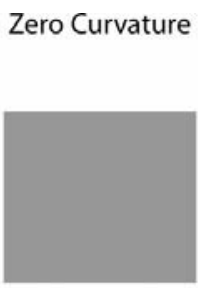

Uniform Growth
Negative Curvature

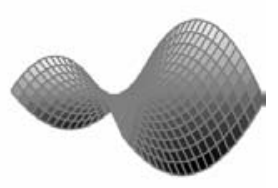

Increased Differentia Growth at Edges
Fig. 1. Differential growth produces either positive or negative curvature.

patch grows at a uniform rate, the resultant surface will be flat; if the patch has a differential growth component such that its edges develop more slowly than the center, then the resultant surface will be spherical (either concave or convex), otherwise referred to as positively curved; if the edges develop faster than the center, the resultant surface will be saddle-shaped, otherwise referred to as negatively curved $^{12,13}$ (see Fig. 1). The more extreme the differential growth gradient, the greater the resultant intrinsic curvature.

Intrinsic curvature is, as its name suggests, an intrinsic property of the surface itself and cannot be removed from it without tearing or deforming the surface - (think of trying to flatten a football, the intrinsic curvature of its surface means that it cannot be mapped to a surface with a different intrinsic curvature, e.g. a flat plane which has no intrinsic curvature). The cerebral cortex is both intrinsically and extrinsically curved. ${ }^{14}$ Extrinsic curvature (or mean curvature), is curvature that arises from the mechanical folding of the surface, and as such is not a property of the surface itself, but rather of how it is embedded in three-dimensional space. For example, a crumpled piece of paper has many curves in it, but these curves can be removed to restore the paper to its original flat appearance. Thus we can say that the folded paper has extrinsic, but not intrinsic curvature.

The degree of intrinsic curvature is proportional to the degree of differential expansion; bigger differences in expansion across the surface give rise to greater degrees of intrinsic curvature. Differential expansion, if present, increases as expansion increases, i.e. as the magnitude surface area increases. This is analogous to changes in cortical gyrification, which also increase as surface area increases. Importantly, differential (non-uniform) rates of surface expansion, as we have noted above, may give rise to uneven distributions of neurons and an increase in the proportion of short connections. Since it also gives rise to intrinsic curvature, it follows that measures of intrinsic curvature contain information about length distributions of tangential cortical connections. This is the essence of our hypothesis.

Although a mathematical concept,${ }^{12}$ the connection between differential development and intrinsic curvature has previously been established in biological studies. It has been demonstrated that the differential growth of leaves produces intrinsic curvature, ${ }^{13}$ while a similar effect was noted in plant roots. ${ }^{15}$ However, measurements of cortical intrinsic curvature have not commonly been assessed in structural studies of the brain. This is likely due, in no small part to its subtle nature compared to the rather more striking, and easily measurable, patterns of gyrification and cortical thickness.

One of the first studies to investigate cortical intrinsic curvature was based on a cortical reconstruction of the Talairach atlas, and implicitly confirmed the prevalence of intrinsic curvature by demonstrating that the mean geodesic (smallest path length between two points on a surface, a function of intrinsic curvature) was less than its total surface area would predict. ${ }^{2}$ Other studies explicitly measured the intrinsic curvature of the cortex at a millimeter-scale and showed that it has both negative and positive curvature. ${ }^{16}$ More recently, the intrinsic curvature of the cortical surface, again measured at a millimeter-scale, was explicitly rendered, ${ }^{17}$ and a qualitative examination of the surface revealed it to be predominantly negatively curved. The average magnitude of intrinsic curvature measure at this scale is about $0.06 \mathrm{~mm}^{-2}{ }^{18}$

Thus far we have discussed the implication of differential expansion on connectivity and morphology separately. In order to explore the possibility of this link empirically, we contrasted cortical intrinsic curvature between humans and chimpanzees. We hypothesized that if morphology and connectivity are linked then there should be quantifiable differences in intrinsic curvature between the species, over and above those attributable to differences in surface area, and that these differences should conform to theoretical expectations of connectivity differences between the species. 
We chose humans and chimpanzees as a contrast based on the strong theoretical arguments and empirical evidence of connectivity differences. Large brains are not simply scaled-up versions of small brains. ${ }^{19}$ Rather, theoretical considerations predict that increasing brain size results in proportionately fewer long-range connections. ${ }^{20}$ As discussed in Ref. 21, if a fixed percentage of connectivity was maintained, then the volume of connections would increase exponentially as brain size increased. Without a trend towards increasing modularity, the overall increase in distance of all connection lengths in larger brains would result in a conduction delay, which is inefficient. ${ }^{11,22}$ Empirical studies have supported this and demonstrated, for example, that inter-hemispheric connectivity via the corpus callosum is reduced in larger primate brains, while the intra-hemispheric connectivity is augmented..$^{23,24} \mathrm{In}$ a similar manner but at a smaller scale, the proportional extent of horizontal cortical connections in the primary visual cortex, $\mathrm{V} 1$, is decreased in macaque monkeys (V1 of $1200 \mathrm{~mm}^{2}$, Ref. 25) compared to tree shrews (V1 $120 \mathrm{~mm}^{2}$, Ref. 26).

In terms of cortical architecture, humans have a lower neuronal density and increased horizontal spacing between minicolumns than chimpanzees and other primates. ${ }^{27,28}$ This implies that, on average, in accord with the theory outlined above, there should be a greater uneven spatial distribution in the lower density cortex (humans), and hence (as argued above) more skew towards shorter connections. Thus, although the absolute connection lengths in chimpanzees may be shorter, they will have proportionately more "long" connections. The reduced neuronal density in humans will also impact on differential growth which arises from forces internal to the surface itself. Hence differences in cortical architecture between humans and chimpanzees will also manifest as differences in differential growth, with the lower density cortex experiencing greater degrees of differential growth.

We have thus far discussed two factors which we propose impact on the degree of differential growth, namely magnitude surface area, and cortical architecture. In a comparison between humans and chimpanzees we expect that humans will have relatively more differential growth due to their larger surface areas, as well as reduced neuronal density. After correction for surface area, differences in the distribution of intrinsic curvature values (taken as a proxy for differential growth) should solely reflect differences in underlying architecture. We believe these changes may ultimately be related to cortico-cortical connectivity.

In summary, although the relationships between intrinsic curvature and differential development, and between differential development and cortical connection lengths have been independently explored, we believe that these hitherto separate strands may usefully be brought together. We postulate that differential development simultaneously affects the relative lengths of cortico-cortical connections, and the degree of intrinsic curvature and, thus, intrinsic curvature, which is quantifiable, may serve as a useful marker for tangential cortical connectivity distributions. If the degree of intrinsic curvature reflects differential development and is related to cortical connectivity, then humans should have higher degrees of intrinsic curvature over and above the magnitude of cortical surface area.

\section{Methods}

\subsection{Subjects \& $M R$ acquisition parameters}

We obtained three-dimensional T1-weighted MPRAGE magnetic resonance (MR) images for ten chimpanzees and ten humans using a Siemens 3 Tesla (T) Trio MR system. Chimpanzee images were acquired at Yerkes National Primate Research Centre (YNPRC) in Atlanta, Georgia, US. Human images were acquired at the Magnetic Resonance and Image Analysis Research Centre (MARIARC) at the University of Liverpool, UK. The MR sequences used for acquisition of images are shown in Table 1. Acquisition parameters were made to be as similar as possible for humans and chimpanzees. The field of view was naturally larger in humans owing to greater head size. Furthermore, we were unable to replicate the $0.6 \mathrm{~mm}$ voxel resolution in the slice thickness direction in humans that was used for the chimpanzees due to the presence of multiple artefacts. Reducing the voxel resolution to $1.0 \mathrm{~mm}$ in this direction in humans resulted in an acquisition time of 12 minutes, which contrasted to the 36 minute chimpanzee scan. However, the inplane voxel resolution was $0.6 \mathrm{~mm} \times 0.6 \mathrm{~mm}$ for both humans and chimpanzees, resulting in a very similar 
Table 1. MRI acquisition information for study images.

\begin{tabular}{lll}
\hline & \multicolumn{1}{c}{ Chimpanzee } & \multicolumn{1}{c}{ Human } \\
\hline$n$ & 10 & 10 \\
Male/Female & $4 / 6$ & $6 / 4$ \\
Hardware & $3 \mathrm{~T}$, Siemens trio, CP head coil & $3 \mathrm{~T}$, Siemens trio, CP head coil \\
Sequence & MPRAGE T1-weighted & MPRAGE T1-weighted \\
TR & $2300 \mathrm{~ms}$ & $2300 \mathrm{~ms}$ \\
TE & $4.4 \mathrm{~ms}$ & $4.4 \mathrm{~ms}$ \\
TI & $1100 \mathrm{~ms}$ & $1100 \mathrm{~ms}$ \\
Flip angle & 8 & 8 \\
NEX & 3 & 1 \\
FOV & $200 \mathrm{~mm} \times 200 \mathrm{~mm}$ & $200 \mathrm{~mm} \times 200 \mathrm{~mm}$ \\
In-plane resolution & $0.625 \mathrm{~mm} \times 0.625 \mathrm{~mm}$ & $0.625 \mathrm{~mm} \times 0.625 \mathrm{~mm}$ \\
Slice thickness & $0.6 \mathrm{~mm}$ & $1 \mathrm{~mm}$ \\
Data matrix size & $320 \times 320$ & $320 \times 320$ \\
Slices & 192 & 192 \\
Time & $36 \mathrm{minutes}$ & $12 \mathrm{minutes}$ \\
\hline
\end{tabular}

between-tissue contrast. All humans provided written consent to participate in this study, which had local ethics committee approval. For the chimpanzee scans, subjects were first immobilized by ketamine injection $(10 \mathrm{mg} / \mathrm{kg})$ and subsequently anaesthetized with propofol $(40-60 \mathrm{mg} / \mathrm{kg} / \mathrm{h})$ following standard procedures at the YNPRC. Subjects were then transported to the MRI facility and remained anaesthetized for the duration of the scans as well as the time needed to transport them between their home cage and the imaging facility (total time $\sim 2 \mathrm{~h}$ ). Subjects were placed in the scanner chamber in a supine position with their head fitted inside the human-head coil.

\subsection{FreeSurfer cortical reconstruction}

Cortical reconstructions were generated using the software FreeSurfer (http://surfer.nmr.mgh. harvard.edu/, see Refs. 29-32). The FreeSurfer program was specifically developed for cortical reconstruction. In brief, raw image data voxels are subsampled to voxels of side $1 \mathrm{~mm}^{3}$. After that the data are normalized for intensity, RF-bias field inhomogenities are modeled and removed, followed by skull-stripping. The cerebral white matter is identified based on a linear combination of voxel intensities and local geometric information. After white matter segmentation, the hemispheres are separated from each other and non-cerebral structures. Finally the white matter volume is tessellated and deformed to produce an accurate and smooth representation of the grey-white interface. Because the radius of curvature and the thickness of the cortex are greater than the size of the MR voxels, trilinear interpolation of the surface is not limited by the voxel dimensions of the original data, and hence surfaces may be computed at a sub-voxel scale. ${ }^{31}$

Although the FreeSurfer process is specifically designed for the processing of human data, nonetheless it has previously been used to reconstruct cortical surfaces of non-human primates. ${ }^{33}$ However, in order to ensure accurate surface reconstructions, special attention is required to apply the process to non-human primates. FreeSurfer is optimized to work on voxels conformed to $1 \mathrm{~mm}^{3}$, in a data matrix $256 \times 256 \times 256$. As such the processing of high-resolution data, such as that acquired for this study can be problematic. In order to circumvent such problems, and ensure the most time-efficient and accurate surface reconstructions, the data for humans and chimpanzees were conformed to the expected FreeSurfer dimensions.

Because data were acquired at slightly different resolutions and on different MR scan systems, stringent quality control was applied to ensure the accuracy of the surface reconstruction for each individual. Every slice through each volume was visually inspected to check the accuracy of both the pial and white matter surfaces. Inaccuracies in segmentation and reconstruction were manually corrected, and surfaces recomputed. 


\subsection{Measuring curvature}

In talking about curvature, it is important to be specific as to what is meant. For example, in the commonly held understanding of curvature, we may say that the surface of a sphere and a cylinder are both curved surfaces, however investigating further we can make distinctions between these two surfaces which demonstrate that the nature of their curvature is quite different.

Consider how curvature is measured: curvature is defined at every point on a line or surface. At each point on a line, the curvature is measured as the inverse of the radius of the osculating circle $(c=1 / r)$ (see Fig. 2). Thus if we were to slice through both a sphere and a cylinder to create a line-profile of the shape, we would in each case measure a non-zero curvature. Now consider measuring curvature of a surface: at every point on the surface there is an infinite number of directions through which we can measure the curvature. Gauss showed that, despite this, there are always two directions which produce a maximum and a minimum value of curvature, and these directions are always orthogonal to each other (called the principals of curvature $\left.\left(c_{1}, c_{2}\right)\right)$. Gauss thus described the curvature at a point on a surface as the product of the principal curvature measured in each of these directions $\left(K=c_{1} \times c_{2}\right)$. This type of curvature is called intrinsic or Gaussian curvature, and is a function of the surface itself.

If we return to our cylinder and sphere, we can now begin to differentiate between the curvatures of each of these surfaces. For example, in the case of the cylinder, it is easy to appreciate that the maximum curvature is perpendicular to the axis of the cylinder, while the minimum curvature is parallel to it. In the case of the latter, the radius of curvature is infinite, given that the axis is a straight line. This means that at every point on the cylinder surface, the intrinsic curvature is zero, implying that it is homomorphic with a flat plane. We can confirm this by considering the lack of distortion to the surface if we unfold a cylinder to lie flat on a plane surface. In contrast, for each direction on a spherical surface, there is a non-zero value to each of the radii of curvature (actually they are identical). This means that the surface cannot be flattened without distortion.

The distinction between folding-based curvature (mean curvature), and intrinsic surface-curvature is subtle and it is not always possible to appreciate visually, especially when the two co-exist in the same surface, as is the case with the cerebral cortex, however some work has been done to contrast these two parameters in the visual cortex. ${ }^{34}$ To illustrate the difference between folding and intrinsic curvature, the mean curvature, $\mathrm{H}$, which is a measure of folding, and the intrinsic curvature are mapped on to a cortical reconstruction in Fig. 2. Mean curvature is calculated as the average of the principal curvatures (formally it is defined as the trace of the Hessian matrix, while intrinsic curvature is the determinant). Thus depending on how they are combined, the principal curvatures can illustrate the folding of the cortex (gyri and sulci, as convex or concave folds respectively), or the intrinsic curvature of the cortex (both positive and negative intrinsic curvature).

Mathematically, curvature is measured at each point on a surface. In the FreeSurfer process, this is approximated through intrinsic curvature measures at each vertex of the surface reconstruction using the principles of the Gauss-Bonnet scheme (see Fig. 2(d)): On a flat surface, the interior angles of a triangle sum to $180^{\circ}$. However, as discovered by Riemann, on a curved surface this does not hold, and the sum of the interior angles are greater or less than $180^{\circ}$ for positive or negative curvature respectively, the surfeit or deficit depending on the degree of intrinsic curvature. These relationships are the basis for intrinsic curvature calculations on the cortical surface: if the area of a vertex (defined as the area of triangles which define the vertex) is convex or concave (as is the case for a positively curved surface), then the surrounding angle is less than $360^{\circ}$. If the area is flat, then the angle is $\pm 360^{\circ}$, whereas if the area is saddle-shaped (negatively curved), the angle is greater than $360^{\circ}$. The intrinsic curvature of the vertex is calculated as the surfeit or deficit of the vertex angle divided by one third the sum of the vertex areas (Eq. (1)). ${ }^{35}$

$$
K=\frac{2 \pi-\sum_{i} \theta_{i}}{\frac{1}{3} \sum_{i} A_{i}}
$$

where $\theta_{i}$ is the angle subtended by $i$ th vertex, and $A_{i}$ is the area of $i$ th vertex (the sum of areas of triangle surrounding the vertex). (Further details of curvature calculations per vertex are outlined in Ref. 17.) The Gauss-Bonnet scheme has been demonstrated 


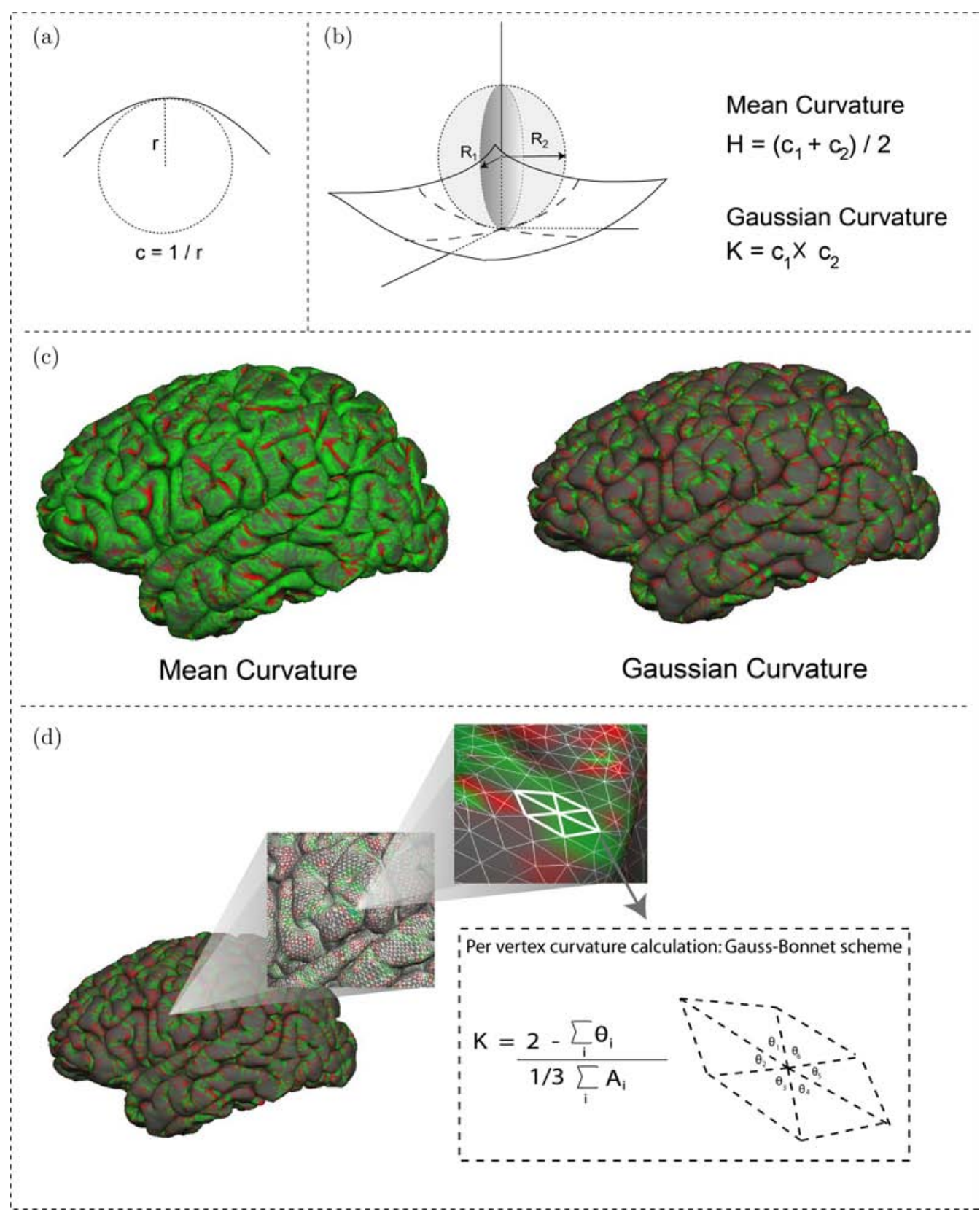

Fig. 2. (a) The curvature, c, at a point on a line is given as the inverse of the radius of the osculating circle at that point. (b) For a surface, the curvature at a point is the function of the principal curvatures at that point, where the principal curvatures are always orthogonal to each other. The mean curvature, a function of how the surface is embedded in space, is the average of the principal curvatures, while the Gaussian curvature, which is intrinsic to the surface, is calculated as the product of the principal curvatures. (c) The principal curvatures for each point on a cortical surface reconstruction may be combined to generate a map of mean curvature or Gaussian curvature. The mean curvature clearly follows the familiar morphology of the cortex, with convex regions (gyri) in green, and concave regions (sulci) in red. The pattern of Gaussian curvature is of a much higher spatial frequency and does not follow the larger-scale morphological features of cortical folds. For Gaussian curvature, positive curvature is red and negative curvature is green. These images demonstrate how folding is distinct from intrinsic curvature. (d) For a surface reconstruction the Gaussian curvature is calculated per vertex based on the Gauss-Bonnet scheme (see Eq. (1)). 
to be optimal for measurements of Gaussian curvature based on surface tessellations in comparison with other commonly applied methods. ${ }^{35}$ Intrinsic curvature values were measured on the pial surface for each subject.

\subsection{Reliability of curvature measures}

\subsubsection{Filtering}

Despite stringent quality control measures to ensure accurate surface reconstructions, surfaces may yet be hampered by single voxel errors. As discussed previously, ${ }^{17}$ the limiting scale at which curvature may be reliably calculated is defined by an osculating sphere with radius one-half the diagonal of the voxel. In the FreeSurfer process all data are sub-sampled to a unit side of $1 \mathrm{~mm}$, giving a limit of resolution of $\sqrt{ } 2 / 2 \mathrm{~mm}$, equivalent to a radius of curvature of $1.41 \mathrm{~mm}^{-1}$ (see Fig. 3). Although surfaces are reconstructed at a sub-voxel scale (being continuous), this value represents the threshold of curvature beyond which values are not reliable.

In order to filter out unreliable curvature values, the intrinsic and mean curvature values for each vertex are calculated using the Gauss-Bonnet method described above. From there the principle curvature values are derived. At this point the limiting threshold is applied to both principal curvatures

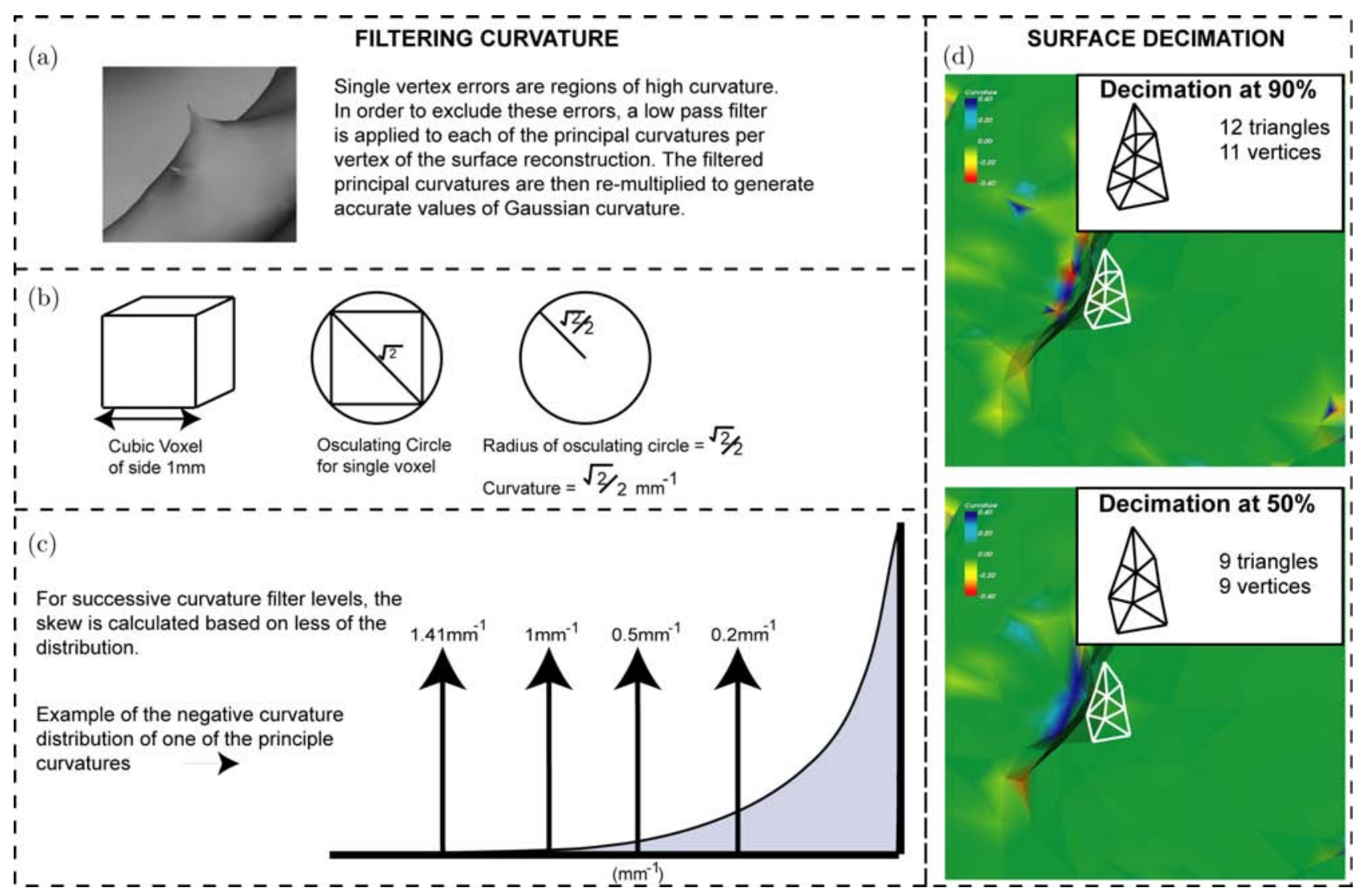

Fig. 3. (a) Single vertex errors sometimes occur in surface reconstructions despite manual editing. These result in vertices of very high curvature. In order to remove these, Gaussian curvature values per vertex are resolved into their constituent principal curvatures and a low-pass filter applied. Subsequently the filtered principal curvatures are re-multiplied to produce a filtered Gaussian curvature per vertex of the surface reconstruction. (b) Different levels of filtering are applied. At the lowest level, the low-pass filter value is defined by the limiting scale at which curvature may be reliably calculated. In FreeSurfer, this is defined by the inverse of the radius of an osculating sphere half the diameter of the voxel. (c) Because the cerebral cortex is smooth, less highly curved shapes are more likely to reflect the shape of the cortex, thus different filter levels are applied to the curvature distribution. (d) An example of the impact of surface decimation at the $90 \%$ and $50 \%$ level. As the surface is down-sampled, the number of vertices and triangles are decreased. 
from which the intrinsic curvature value is subsequently derived. Principal curvature values that are more extreme than the filter threshold are set to zero. Thereafter the filtered principal curvatures for each vertex are multiplied to produce filtered intrinsic curvature values for each vertex in the cortical reconstruction, and hence a filtered distribution of intrinsic curvature values for each subject.

The filter level $\left|1.41 \mathrm{~mm}^{-1}\right|$ represents the limiting resolution by which we can rely on principal curvature measures, however this resolution does not preclude the possibility that data may be affected by surface reconstruction errors which are greater than the dimensions of a single voxel and yet not removed by manual editing. Because of this, several different filter levels are applied to principal curvature calculations, such that principal curvature values become less extreme and hence are more likely to follow the smooth surface of the cortex.

Four different filter levels were applied to each principal curvature calculation, i.e. $c_{1}, c_{2}: \mid 1.41$ $\mathrm{mm}^{-1}|| 1 \mathrm{~mm}^{-1}|, \quad| 0.5 \mathrm{~mm}^{-1} \mid$ and $\left|0.2 \mathrm{~mm}^{-1}\right|$. At each stage, vertices with principal curvature values exceeding these levels were set to zero and hence the intrinsic curvature value for that vertex was excluded from further distribution analysis (see Fig. 3). Per vertex, principal curvature values that survive each level of filtering are multiplied together to produce a filtered Gaussian curvature for that vertex.

Because chimpanzees have smaller brains and hence higher average curvature values (irrespective of subtle shape differences), the same low-pass filter level applied to unconformed human and chimpanzee data sets will remove a higher proportion of the chimpanzee curvature distribution. To compensate for this, we applied the same filter levels to conformed data (see Sec. 2.2). This had the effect of producing equivalent percentages of curvature distributions for each filter level in a comparison across the species.

\subsubsection{Surface decimation}

Vertex-based measures of intrinsic curvature may be susceptible to artifacts due to local surface quantization effects such as varying the number of vertex neighbors. As an additional test of the reliability of FreeSurfer cortical curvature values, we applied an algorithm which decimated the cortical reconstruction. ${ }^{36}$ Decimation has the effect of down-sampling the number of vertices in the surface reconstruction, while maintaining as far as possible fidelity to the original shape of the cortical surface. In this experiment we decimated the reconstructions per subject to $90 \%, 75 \%$ and $50 \%$ of the original number of vertices. This had the effect of increasing the size of surface triangles, as well as varying the number of neighbors per vertex (see Fig. 3). Curvature analysis was carried out as described above for each of these levels.

\subsection{Data analysis}

As discussed previously, we postulate that differential growth introduces an additional component to the overall forces determining length. Our theory predicts that humans will have proportionately more short-range connectivity than chimpanzees, hence we predict that the distribution of human curvature values will be skewed towards more extreme curvature values reflecting more short-range connectivity due to this differential surface growth component.

Because we are interested in the shape of the curvature distribution rather than its average value, we quantified the skew of the curvature distribution per subject, and averaged this over species. Skew, $S$, is defined as follows (Eq. 2)

$$
S=\frac{1}{n} \sum_{i}^{n}\left[x_{i}-\bar{x}\right]^{3} /\left(\frac{1}{n} \sum_{i}^{n}\left[x_{i}-\bar{x}\right]^{2}\right)^{3 / 2}
$$

where $n$ is the number of intrinsic curvature values (equal to the number of vertices in the surface reconstruction), and $x_{i}$ is the value of Gaussian curvature for the $i$ th vertex. The skew of a distribution is a dimensionless measure of how far from symmetry it is. As reported in Ref. 17, the distributions of positive and negative cortical intrinsic curvature are weighted towards zero. Zero curvature represents an absence of differential growth (a flat surface). As the component of differential growth increase, it affects the curvature distribution, skewing it away from zero. In accordance with our theory, we propose that this reflects an increase in the proportion of short connections. Thus the less skewed the curvature distribution (the more it is weighted away from zero curvature), the greater the influence of differential growth and the greater the proportion of short-range connections. 


\section{Results}

\subsection{Average values}

The average cerebral volume for humans and chimpanzees derived from cortical reconstructions was $723 \mathrm{~cm}^{3}( \pm 76)$ and $421 \mathrm{~cm}^{3}( \pm 37)$ respectively. These results are in good experimental agreement with similar studies. ${ }^{37}$ The average surface area per hemisphere for humans and chimpanzees was $681 \mathrm{~cm}^{2}$ $( \pm 64)$, and $314 \mathrm{~cm}^{2}( \pm 24)$ respectively.

For humans the average intrinsic curvature values for negative and positive curvature were $-0.038 \mathrm{~mm}^{-2}$ and $0.033 \mathrm{~mm}^{-2}$ respectively. For chimpanzees, the average curvature was $-0.018 \mathrm{~mm}^{-2}$ and $0.016 \mathrm{~mm}^{-2}$ respectively,

Finally, the average percentage of vertices with negative curvature was $55 \%( \pm 0.4)$ and $56 \%( \pm 1.7)$ for humans and chimpanzees respectively. The corresponding percentage area for negative curvature was $60 \%( \pm 3)$ for both humans and chimpanzees, demonstrating that the pial surface is predominantly negatively curved for both species. This result is in keeping with previous reported findings. ${ }^{2,17}$

\subsection{Cortical intrinsic curvature distributions}

The spatial distributions of cortical curvature for chimpanzees and humans at the $\left|1.41 \mathrm{~mm}^{-1}\right|$ filter are illustrated in Fig. 4. Empirically, the distributions for both positive and negative intrinsic curvature are weighted towards zero, and are similar to those previously published. ${ }^{16,17}$

Skew analysis of the curvature distributions for humans and chimpanzees (corrected for surface area) revealed significant differences between the species (Fig. 5). For positive and negative curvature, humans are significantly less skewed (less weighted towards zero) than chimpanzees. This finding is robust across all filter levels (Table 2).

This result is in agreement with our hypothesis stated at the outset, that humans have a greater degree of intrinsic curvature (taken as a proxy for differential growth) than chimpanzees, over and above surface area differences. It is our hypothesis that this result reflects a change in the proportion of short over long connections between the species.

\subsection{Reliability of measures}

The effects of different curvature filter levels are reported in Table 2. For each filter level, the percentage of vertices contributing to intrinsic curvature measures were $81 \%, 74 \%, 50 \%$ and $12 \%$ for filters $\left|1.41 \mathrm{~mm}^{-1}\right|,\left|1 \mathrm{~mm}^{-1}\right|,\left|0.5 \mathrm{~mm}^{-1}\right|$ and $\left|0.2 \mathrm{~mm}^{-1}\right|$ respectively. There were no significant differences between the species for any of the filter levels, except at the $\left|0.2 \mathrm{~mm}^{-1}\right|$ level, which may be

\section{- Positive Curvature}

- Negative Curvature

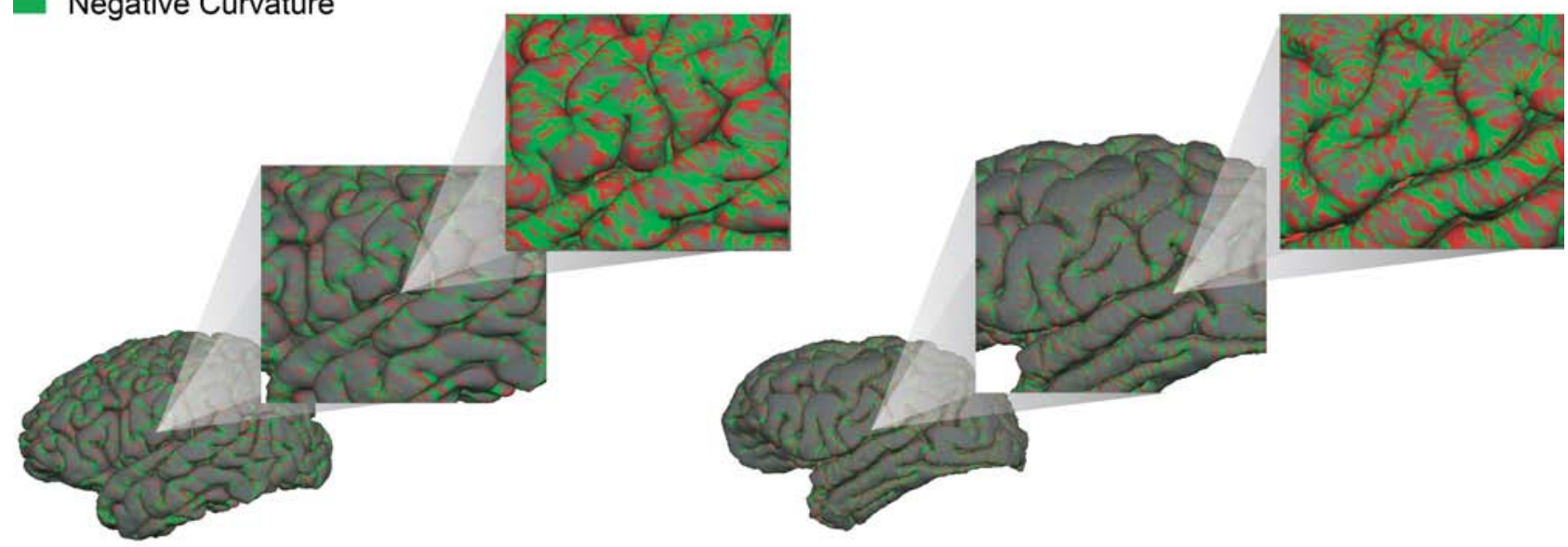

Human

Chimpanzee

Fig. 4. Gaussian curvature for humans and chimpanzees for the pial surface. In each case, the high spatial frequency pattern of the Gaussian curvature is evident. 

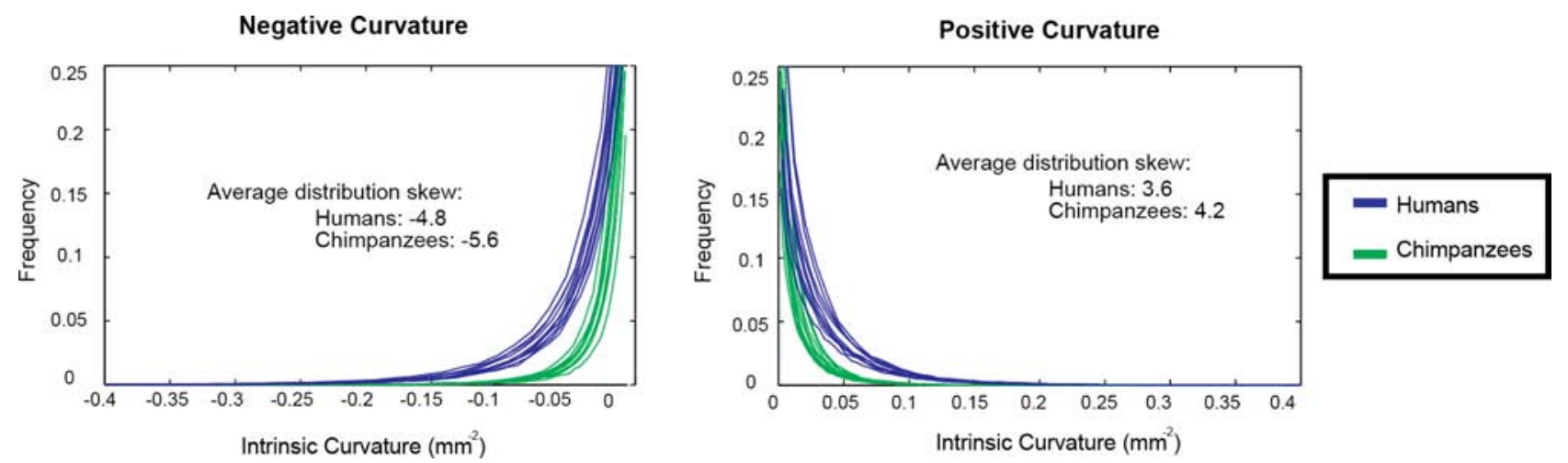

Fig. 5. Distributions of positive and negative cortical intrinsic curvature for humans (blue) and chimpanzees (green) based on the cortical surface. In each case the magnitude of skew of the curvature distribution is less in humans.

Table 2. Average values of the skew of the intrinsic curvature distributions (both positive and negative), broken down by species and curvature filter level. In agreement with theoretical predictions, for both positive and negative curvature, chimpanzees are more skewed than humans. This pattern is consistent across all filter levels.

\begin{tabular}{llccr}
\hline & & Humans & Chimpanzees & $p$-value \\
\hline Negative & $|1.41 \mathrm{~mm}|$ & -4.8 & -5.6 & 0.04 \\
Curvature & $|1 \mathrm{~mm}|$ & -3.8 & -4.8 & 0.02 \\
Skew & $|0.5 \mathrm{~mm}|$ & -2.6 & -3.3 & $<0.01$ \\
& $|0.2 \mathrm{~mm}|$ & -1.9 & -2.3 & 0.03 \\
Positive & $|1.41 \mathrm{~mm}|$ & 3.6 & 4.2 & 0.05 \\
Curvature & $|1 \mathrm{~mm}|$ & 3.2 & 3.6 & 0.01 \\
Skew & $|0.5 \mathrm{~mm}|$ & 2.7 & 3 & $<0.01$ \\
& $|0.2 \mathrm{~mm}|$ & 2.7 & 3.2 & $<0.01$ \\
\hline
\end{tabular}

due to the differences in skew of the distributions. For all filter levels, humans are both less positively and less negatively skewed in comparison to chimpanzees, in keeping with theoretical predictions.

The effects of surface decimation are reported in Table 3. Three different levels of surface

Table 3. Results of curvature skew analysis for different decimation levels.

\begin{tabular}{|c|c|c|c|c|}
\hline & $\begin{array}{c}\text { Decimation } \\
(\%)\end{array}$ & Humans & Chimpanzees & $p$-value \\
\hline Negative & 90 & -7.2 & -7.6 & 0.07 \\
\hline Curvature & 75 & -6.9 & -7.5 & 0.01 \\
\hline Skew & 50 & -6.2 & -6.8 & $<0.01$ \\
\hline Positive & 90 & 7.5 & 7.6 & 0.44 \\
\hline Curvature & 75 & 7.1 & 7.5 & 0.01 \\
\hline Skew & 50 & 6 & 6.6 & $<0.01$ \\
\hline
\end{tabular}

decimation were applied. The average vertex area was $0.98 \mathrm{~mm}^{-2}, 1.04 \mathrm{~mm}^{-2}, 1.16 \mathrm{~mm}^{-2}$, and $1.45 \mathrm{~mm}^{-2}$ for the original, and $90 \%, 75 \%$ and $50 \%$ decimations levels respectively. For each decimation level, humans have less curvature skew compared to chimpanzees, in keeping with expectations. These results demonstrate the robustness of curvature values to artifacts of surface reconstruction.

\section{Discussion}

\subsection{Overview}

This experiment examines the prediction that the human brain should show a greater degree of intrinsic curvature than that of the chimpanzee independent of surface area. This prediction is based on the proposed relationship between intrinsic curvature and tangential cortical connection length distributions, a link based on the premise that differential surface expansion has an impact on each.

The approach described unites two separate observations: on the one hand differential rates of tangential surface development introduce intrinsic curvature, with greater rates of differential expansion resulting in higher degrees of curvature. Commensurate with this, differential expansion results in greater disparity of inter-neuronal distances, skewing the length distribution to favor proportionately more short-range cortico-cortical connections. Thus, because differential development affects both the distribution of curvature values and the distribution of tangential connection lengths, we suggest that it is possible to adopt measures of the former as markers of the latter. 
More specifically, in the absence of differential development, there will be a certain distribution of connection lengths. As the influence of differential development increases, the original length distribution will be skewed to proportionately more short connections. Here, we compare this imposed skew across species, and make inferences about the relative proportions of short-to-long connections. We highlight that, just as in conventional network analysis, the current method does not quantify absolute connection lengths, but rather the relative difference in the proportions of short connection lengths between humans and chimpanzees. The a priori prediction was that the two species would have distinct connection length distributions, over and above the disparity of magnitude in surface area. We propose that this reflects a difference in cortical architecture, with the larger brains of humans characterized by a more uneven spatial distribution and hence a preponderance of short connections, as well as increased intrinsic curvature. The results of our analysis demonstrate agreement with this prediction.

The interpretation of intrinsic curvature, and its impact on the cortical surface is dependent on the scale at which it is measured. For example, at a gross scale, the shape of the whole brain is quasi-spherical, suggesting a large-scale positive intrinsic curvature, complementing the shape of the cranium. At lower scales, such as the centimeter-scale employed in Ref. 2, or the millimeter-scale in Ref. 17, the majority of the cortex appears to be negatively curved. We propose that measures of cortical intrinsic curvature at a millimeter-scale may be related to the differential tangential development of cortical connections at that scale. Crucially, when we refer to cortico-cortical connections we mean intrinsic (horizontal) axonal processes that are confined to the cortical grey matter, which can extend up to several millimeters. ${ }^{38}$ We do not mean extrinsic (white matter) connections such as U-fibers, or intra and inter hemispheric connections. In other words, the connectivity we refer to is at a mesoscopic scale of a few millimeters and is tangential to the cortical surface.

\subsection{Reliability}

It is noteworthy that two of the observations made here pertaining to intrinsic curvature are compatible with those made in previous studies: the high-frequency pattern of intrinsic curvature, ${ }^{16,17}$ and the predominance of negative intrinsic curvature. ${ }^{2,17}$ Further, the values of intrinsic curvature we report are comparable to previous findings. ${ }^{18}$

One of the limitations of this study is the fact that data were not acquired on the same scanner, however every attempt was made to match as closely as possible the scanning parameters between centers. In addition intensive quality control was applied to the surface reconstructions for each subject. Where errors in reconstruction were noted (e.g. due to poor contrast between tissue classes), manual edits were carried out. Because inaccuracies are corrected based on two-dimensional slices, and not the three-dimensional surface representation, this type of intervention is not biased with respect to the measurement of intrinsic curvature (which is not measurable on two-dimensional line profiles, see Sec. 2.3, Measuring Curvature). By inspecting the cortical reconstruction of each subject, we aimed to reduce, as far as possible, discrepancies in accuracy that may have arisen from differences in data acquisition. As a further control, we applied a series of curvature filters to remove values arising from single vertex errors that were not correctable with manual edits. Finally we tested the reliability of curvature values by applying a series of surface decimation levels to the cortical mesh of each subject to ensure that curvature values were representative of cortical morphology and not surface quantization effects. The results of our validity experiments demonstrate the reliability of our data and the robustness of the methods used.

\subsection{Theoretical considerations}

Theoretically intrinsic curvature is very interesting and the distinction between negative and positive intrinsic curvature is potentially useful. The possible advantages of negative and positive curvature were originally contrasted in Ref. 2. At the heart of the discussion is the distinct effects each shape has on angles and lengths along the surface. In the case of spherical or positive intrinsic curvature, angles and distances are increased relative to Euclidean geometry, whereas for negative intrinsic curvature, angles and distances on the surface are reduced compared to Euclidean geometry. For example, consider a fixed point, $P$, attached to which is a length of rope, $r$. 
If one were to walk around the fixed point, while keeping the rope completely taut, the distance traversed would be $2 \pi r$ if the surface were flat. Now consider if the surface were spherical, the circumference traversed would be less than that measured on the flat surface, as the rope, although taut, would now be effectively "shortened" in reach due to the intrinsic curvature of the surface. Conversely, if the surface were hyperbolic in shape, as if the point $P$ were on a valley floor, the measured circumference would be greater than $2 \pi r$ as the rise and fall of the surface topology would act to lengthen the distance required for a revolution. Thus, in order to produce an equivalent circumference to that measured on the flat plane, the size of the spherical area would have to be increased, whereas the size of the hyperbolic area would have to be decreased. From this reasoning we can postulate that the predominantly negative nature of cortical intrinsic curvature may introduce an increased level of efficiency to the cortical surface, in that, for a given circumference of a patch of cerebral cortex, a smaller area is covered than would be the case for a flat or spherical patch. This means that there is a greater circumference for a given radius compared to a positively curved patch or a circle on a flat surface. The impact of this was discussed in Ref. 2, where it was suggested that this may imply that a negatively curved cortical patch could facilitate more inter-regional (tangential) connectivity and hence be superior for inter-area wiring. Additionally it was suggested that because the average geodesic on a negatively curved surface is less than on a positively curved or flat surface, it may be more efficient for intra-area wiring also. Given these considerations, the preponderance of negative versus positive intrinsic curvature of the cortical surface at a millimeter-scale may be considered commensurate with the idea that the brain has adapted its shape to be maximally energy efficient. ${ }^{39}$ It is estimated that approximately $50 \%$ of the brain's energy is used to drive signals, ${ }^{40}$ with higher proportions of energy required for cortical grey matter $(75 \%)$ compared to the rest of the brain reflecting the formers greater levels of inter-connectivity. Thus it may be that the brain has evolved a shape that minimizes the energy cost of signal transfer across its surface.

At larger scales, measurements of intrinsic curvature may prove to be very informative regarding the organization and appearance of the cortex. Just as the smoothness of a piece of paper is dependent on the scale at which it is measured, so too is the intrinsic curvature of cortex. That is, the spatial frequency and values of this intrinsic curvature vary over different scales. For example, at the millimeterscale, the cortex has a demonstrably high spatial frequency, independent of the larger-scale patterns of gyri and sulci, whereas at the centimeter-scale it has a lower frequency that follows more closely cortical gyrification features. ${ }^{2}$ Similarly the values of intrinsic curvature measured are dependent on the scale at which they are measured. For example, in this experiment the average intrinsic curvature values of the pial surface for humans (with minimal filtering) were $-0.038 \mathrm{~mm}^{-2}$ and $0.033 \mathrm{~mm}^{-2}$, however at higher sales, we would expect to measure proportionately less sharp intrinsic curvature values. Because intrinsic curvature values are dependent on not only the shape but also the size of the object being measured, it is important, as we have done here, to use dimensionless parameters such as skew when comparing brains of different sizes (For a more complete discussion see Ref. 17).

\subsection{Future work}

The hypothesis proposed here raises several testable predictions, namely that smaller brains will have less intrinsic curvature than larger brains, and that cortices with higher neuronal density will have less differential growth and hence less intrinsic curvature. This may prove useful in clinical studies where higher neuronal density and decreased neuropil have been linked to diseases such as schizophrenia. ${ }^{41,42}$ Further empirical observations of the extent of horizontal connectivity between species, or indeed in different regions of the brain, would be useful to correlate with our predictions for the morphology of the cortex. For example perceptual effects such as the simultaneous tilt illusion, ${ }^{43}$ hypothesized to be a function of horizontal connectivity, should provide an alternative method for generating strong, directional predictions for cortical curvature.

The method proposed here, while suitable for whole brain characterization, may also be adapted to a region-of-interest approach. In particular this may be of interest in studies of disrupted connectivity, which is thought to play a role in diseases such as epilepsy, ${ }^{44}$ or possibly in neurodevelopmental 
disorders, where deviant brain growth trajectories have been linked to abnormalities in cortico-cortical connectivity. ${ }^{45}$

The high spatial frequency pattern of intrinsic curvature is different from that predicted by current theories of gyrification, ${ }^{46}$ which suggest that axonal tension mediates cortical folding, and that differential growth between layers is a consequence, rather than a driver of sulcal/gyral formation. Under this hypothesis, intrinsic curvature should follow a spatial pattern equivalent to the pattern of sulci and gyri, which is not the case. Differential growth between cortical layers, and between the cortex and subcortical white matter has previously been considered as a main component of cortical gyrification, ${ }^{47}$ and further work to explicitly compare extrinsic folding and intrinsic curvature might help resolve the role each plays in gyrification. In particular, analysis of intrinsic curvature at a scale on the order of cortical folds may prove especially useful, given that intrinsic properties of a surface actually constrain the possibly extrinsic properties. $^{2}$

To date connectivity studies using EEG/fMRI/ MRI-based network analysis have focused on largescale, inter-regional (white matter-based) connectivity. However, by measuring intrinsic curvature at the smaller, millimeter-scale, we are able to make inferences about connection length distributions at this scale. We believe that the current approach may offer a novel analysis that is potentially complementary analysis to larger-scale network studies.

\section{Conclusions}

To summarize, in this study we draw a theoretical link between two prior observations: that differential development of the cortical sheet will have an impact on the lengths of tangential cortical connections, and that this differential development will simultaneously affect local cortical morphology and result in intrinsic curvature. We hypothesized that if cortical intrinsic curvature and the length of tangential cortical connections were in fact related, then measures of the former should be able to distinguish between species in a predictable way, based on the theory that larger brains have proportionately more short connections. We demonstrate that, in keeping with predictions, the human brain in comparison to that of the chimpanzee shows a pattern of intrinsic curvature that is commensurate with a greater preponderance of short-range connections. We believe that this novel approach for the characterization, in vivo, of connectivity in the cortical sheet will be complementary to studies evaluating longer range connections, and may prove useful in identifying and understanding functional abnormalities in patient groups.

\section{Acknowledgments}

This work was supported by the Wellcome Trust and the Bernard Wolfe Health Neuroscience Fund, and carried out at the Wellcome/MRC funded Behavioural and Clinical Neurosciences Institute, University of Cambridge. We would also like to thank L. Parr and D. Ginsburg.

\section{References}

1. M. Hofman, On the evolution and geometry of the brain in mammals, Prog. Neurobiol. 32 (1989) 137-158.

2. L. D. Griffin, The intrinsic geometry of the cerebral cortex, J. Theor. Biol. 166 (1994) 261-273.

3. J. T. Eayrs and B. Goodhead, Postnatal development of the cerebral cortex in the rat, J. Anat. 93 (1959) 385-402.

4. S. U. Dani, A. Hori and G. F. Walter (eds.), Principles of Neural Aging, Amsterdam, Elseiver (1972).

5. D. Watts and S. Strogatz, Collective dynamics of 'small-world' networks, Nature 393 (1998) 440-442.

6. M. P. Young, J. W. Scannell, M. A. O'Neill, C. C. Hilgetag, G. A. P. C. Burns and C. Blakemore, Nonmetric multidimensional scaling in the analysis of neuroanatomical connection data and the organization of the primate cortical visual system, Philos. Trans. R. Soc. Lond. B Biol. Sci. 348 (1995) 281308.

7. R. Albert and A. Barabasi, Statistical mechanics of complex networks, Reviews of Modern Physics $\mathbf{7 4}$ (2002) 47-97.

8. D. Bassett and E. Bullmore, Small-world brain networks, Neuroscientist 12 (2006) 512-523.

9. B. He, G. Shulman, A. Snyder and M. Corbetta, The role of impaired neuronal communication in neurological disorders, Curr. Opin. Neurol. 20 (2007) 655660 .

10. S. Achard and E. Bullmore, Efficiency and cost of economical brain functional networks, PLoS Comput. Biol. 3 (2007) e17.

11. G. Buzsáki, C. Geisler, D. Henze and X. Wang, Interneuron diversity series: Circuit complexity and axon wiring economy of cortical interneurons, Trends Neurosci. 27 (2004) 186-193. 
12. P. Todd, Gaussian curvature as a parameter of biological surface growth, J. Theor. Biol. 113 (1985) 63-68.

13. U. Nath, B. Crawford, R. Carpenter and E. Coen, Genetic control of surface curvature, Science $\mathbf{2 9 9}$ (2003) 1404-1407.

14. D. Van Essen and J. Maunsell, Two-dimensional maps of the cerebral cortex, J. Comp. Neurol. 191 (1980) 225-281.

15. A. Chavarra-Krauser, K. A. Nagel, K. Palme, U. Schurr, A. Walter and H. Scharr, Spatio-temporal quantification of differential growth processes in root growth zones based on a novel combination of image seqeunce processing and refined concepts describing curvature production, New Phytologist 177 (2008) 811-821.

16. D. C. Van Essen and H. A. Drury, Structural and functional analyses of human cerebral cortex using a surface-based atlas, J. Neurosci. 17(18) (1997) 7079-7102.

17. R. Pienaar, B. Fischl, V. Caviness, N. Makris and P. Grant, A methodology for analyzing curvature in the developing brain from preterm to adult, Int. J. Imaging Syst. Technol. 18 (2008) 42-68.

18. N. Markis, J. Kaiser, C. Haselgrove, L. J. Seidman, J. Biederman, D. Boriel, E. M. Valera, G. M. Papadimitriou, B. Fischl, A. S. Caviness and D. N. Kennedy, Humans cerebral cortex: A system for the integration of volume- and surface-based representations, NeuroImage 33 (2006) 139-153.

19. K. Im, J. Lee, O. Lyttelton, S. Kim, A. Evans and S. Kim, Brain size and cortical structure in the adult human brain, Cereb Cortex 18 (2008) 2181-2191.

20. J. Kaas, Why is brain size so important: Design problems and solutions as neocortex gets bigger or smaller, Brain and Mind 1 (2000) 7-23.

21. J. L. Ringo, Neuronal interconnection as a function of brain size, Brain Behav. Evol. 38 (1991) 1-6.

22. V. Braitenberg, Brain size and number of neurons: An exercise in synthetic neuroanatomy, J. Comput. Neurosci. 10 (2001) 71-77.

23. J. Rilling and T. Insel, Differential expansion of neural projection systems in primate brain evolution, Neuroreport 10 (1999) 1453-1459.

24. K. Zhang and T. Sejnowski, A universal scaling law between gray matter and white matter of cerebral cortex, Proc. Natl. Acad. Sci. 97 (2000) 5621-5626.

25. K. Rockland and J. Lund, Intrinsic laminar lattice connections in primate visual cortex, J. Comp. Neurol. 216 (1983) 303-318.

26. D. Lyon, N. Jain and J. Kaas, Cortical connections of striate and extrastriate visual areas in the tree shew, J. Comp. Neurol. 401 (1998) 109-128.

27. N. Schenker, D. Buxhoeveden, W. Blaskmon, K. Amunts, K. Zilles and K. Semendeferi, A comparative quantitative analysis of cytoarchitecture and minicolumnar organization in broca's area in humans and great apes, J. Comp. Neurol. 510 (2008) 117-128.

28. K. Semendeferi, E. Armstrong, A. Schleicher, K. Zilles and G. Van Hoesen, Prefrontal cortex in humans and apes: A comparative study of area 10, American Journal of Physical Anthropology 114 (2001) 224-241.

29. A. Dale, B. Fischl and M. Sereno, Cortical surfacebased analysis. I: segmentation and surface reconstruction, Neuroimage 9 (1999) 179-194.

30. B. Fischl, M. Sereno and A. Dale, Cortical surfacebased analysis. II: Inflation, flattening, and a surfacebased coordinate system, Neuroimage 9 (1999) 195-207.

31. B. Fischl and A. Dale, Measuring the thickness of the human cerebral cortex from magnetic resonance images, Proc. Natl. Acad. Sci. 97 (2000) 1105011055.

32. B. Fischl, A. Liu and A. Dale, Automated manifold surgery: Constructing geometrically accurate and topologically correct models of the human cerebral cortex, IEEE Trans. Med. Imaging 20 (2001) 70-80.

33. B. Conway and D. Tsao, Color architecture in alert macaque cortex revealed by fmri, Cereb Cortex 16 (2006) 1604-1613.

34. O. Hinds, J. P. Polimeni, N. Rajendran, M. Balasubramanian, L. L. Wald, J. C. Augustinack, G. Wiggins, H. D. Rosas, B. Fischl and E. L. Schwartz, The intrinsic shape of humand and macaque primary visual cortex, Cerebral Cortex 18 (2008) 2586-2595.

35. T. Surazhsky, E. Magid, O. Soldea, G. Elber and E. Rivlin, A comparison of gaussian and mean curvatures estimation methods on triangular meshes, IEEE International Conference on Robotics and Automation Proceedings (2003), 1021-1026.

36. P. Lindstrom and G. Turk, Fast and memory efficient polygonal simplification, Proceedings of IEEE Visualization (1998), 279-286.

37. H. Haug, Brain sizes, surfaces, and neuronal sizes of the cortex cerebri: A stereological investigation of man and his variability and a comparison with some mammals (primates, whales, marsupials, insectivores, and one elephant), Am. J. Anat. 180 (1987) 126-142.

38. D. Lewis, D. Melchitzky and G. Burgos, Specificity in the functional architecture of primate prefrontal cortex, J. Neurocytol. 31 (2002) 265-276.

39. V. Klyachko and C. Stevens, Connectivity optimization and the positioning of cortical areas, Proc. Natl. Acad. Sci. 100(13) (2003) 7937-7941.

40. S. Laughlin and T. Sejnowski, Communication in neuronal networks, Science 301 (2003) 1870-1874.

41. D. Buxhoeveden, E. Roy, A. Switala and M. Casanova, Reduced interneuronal space in schizophrenia, Biological Psychiatry 47 (2000) 681-683. 
42. L. Selemon and P. Goldman-Rakic, The reduced neuropil hypothesis: A circuit-based model of schizophrenia, Biological Psychiatry 45 (1999) $17-25$.

43. P. Wenderoth and S. Johnstone, The different mechanisms of the direct and indirect tilt illusions, Vision Research 28 (1988) 301-312.

44. S. Sisodiya, Wiring, dysmorphogenesis and epilepsy: A hypothesis, Seizure 4 (1995) 169-185.

45. N. Barnea-Goraly, H. Kwon, V. Menon, S. Eliez, L. Lotspeich and A. Reiss, White matter structure in autism: Preliminary evidence from diffusion tensor imaging, Biological Psychiatry 55 (2004) 323-326.

46. D. Van Essen, A tension-based theory of morphogenesis and compact wiring in the central nervous system, Nature 385 (1997) 313-318.

47. D. Richman, R. Stewart, J. Hutchinson and V. Caviness, Mechanical model of brain convolution development, Science 189 (1975) 18-21. 